Discrete Comput Geom 32:293-308 (2004)

DOI: $10.1007 / \mathrm{s} 00454-004-1099-1$

\title{
The Area Derivative of a Space-Filling Diagram*
}

\author{
Robert Bryant, ${ }^{1}$ Herbert Edelsbrunner, ${ }^{2}$ Patrice Koehl, ${ }^{3}$ and Michael Levitt ${ }^{3}$ \\ ${ }^{1}$ Department of Mathematics, Duke University, \\ Durham, NC 27708, USA \\ bryant@math.duke.edu \\ ${ }^{2}$ Department of Computer Science, Duke University, \\ Durham, NC 27708, USA \\ edels@cs.duke.edu \\ and \\ Raindrop Geomagic, \\ Research Triangle Park, NC 27709, USA \\ ${ }^{3}$ Department of Structural Biology, Stanford University, \\ CA 94305, USA \\ koehl@csb.stanford.edu \\ michael.levitt@stanford.edu
}

\begin{abstract}
The motion of a biomolecule greatly depends on the engulfing solution, which is mostly water. Instead of representing individual water molecules, it is desirable to develop implicit solvent models that nevertheless accurately represent the contribution of the solvent interaction to the motion. In such models, hydrophobicity is expressed as a weighted sum of atomic surface areas. The derivatives of these weighted areas contribute to the force that drives the motion.

In this paper we give formulas for the weighted and unweighted area derivatives of a molecule modeled as a space-filling diagram made up of balls in motion. Other than the radii and the centers of the balls, the formulas are given in terms of the sizes of circular arcs of the boundary and edges of the power diagram. We also give inclusion-exclusion formulas for these sizes.
\end{abstract}

\footnotetext{
* The research of the second, third, and fourth authors was partially supported by the NSF under Grant CCR-00-86013.
} 


\section{Introduction}

In this paper we study questions about three-dimensional conformations of molecules. As common in biology, we model an atom as a ball bounded by a sphere and a molecule as the union of a finite collection of such balls. This union is referred to as the spacefilling diagram of the molecule. Specifically, we study the derivatives of the weighted and unweighted surface area when the atoms are in motion.

Motivation. Bio-molecular simulations provide a platform where theory and experiment can be combined to improve our chance to unravel the complexity of cell functions. While much effort is presently invested in identifying the actors that play a role, there is a growing need to understand the kinetics of their interactions. The sequence or rate of events that occur as molecules transform between their various possible conformations and interact with each other is described by kinetics. Understanding these conformational transitions as well as the role of motions in molecular interactions are the subjects of molecular dynamics studies. Accurate molecular dynamics simulations remain a major challenge, since they involve thousands of degrees of freedom in the molecule of interest, in addition to the need to account for its water environment. Computer simulations that include a large number of water molecules remain the state of the art in this field, but they are inefficient. It is desirable to develop different approaches, in which the effect of the solvent is taken into account implicitly. Such treatment would make it possible to perform simulations covering much longer time intervals, and including much larger molecular systems.

All solvent effects on a molecule can be included in an effective potential, $W=$ $W_{\text {elec }}+W_{\text {np }}$, in which the first term accounts for electrostatic and the second for nonpolar contributions. $W_{\text {elec }}$ is usually represented by continuum electrostatics, for which several semi-analytical approximate treatments have been proposed by Still et al. (1990), Davis (1994), and Schaefer and Karplus (1996). Many solvation models describe $W_{\mathrm{np}}$ as a weighted sum of the solvent exposed or accessible surface area of each atom of the solute; see Eisenberg and McLachlan (1986), Wesson and Eisenberg (1992), and Fraternali and Van Gunsteren (1996). Inclusion of $W_{\mathrm{np}}$ in a molecular dynamics simulation requires the calculation of accurate molecular surface areas, as well as their analytical derivatives with respect to atomic position.

Previous Work. Lee and Richards (1971) define the accessible surface of a protein as the van der Waals envelope of the molecule expanded by the radius of the solvent sphere about each atom center. Computational methods that evaluate the area of this surface can be divided into approximate and exact methods. Most of the approximate methods rely on numerical integration, by representing the surface with a large number of dots; see Shrake and Rupley (1973) and Legrand and Merz (1993). Some of the approximations are analytical but treat multiple overlapping balls probabilistically or ignore them; see Wodak and Janin (1979), Hasel et al. (1988), and Street and Mayo (1998). The first exact analytical methods for computing the accessible surface area were introduced by Connolly (1983) and Richmond (1984). They have been improved in recent years, the focus by von Freyberg et al. (1993) and Fraczkiewicz and Braun (1998) being on computational efficiency and that of Eisenhaber and Argos (1993) and Gogonea and Osawa (1995) being on stability. The idea of using inclusion-exclusion to reduce intersections 
of five or more balls to linear combinations of at most four balls was introduced by Kratky (1978) and by Gibson and Scheraga (1987). Doing the reduction correctly remains however computationally expensive. The Alpha Shape theory solves this problem using Delaunay triangulations and their filtrations, as described by Edelsbrunner (1995). Alpha shapes have been used to compute the surface area and volume of proteins as well as for detecting and measuring cavities in proteins; see Liang et al. (1998).

The distinction between approximate and exact computation also applies to existing methods for computing the derivatives of the surface area with respect to atomic coordinates. Computationally more efficient are the approximate methods implemented in the MSEED software by Perrot et al. (1992) and the SASAD software by Sridharan et al. (1994). The computational efficiency of MSEED is a consequence of ignoring the contribution of internal atoms, at the cost of missing buried cavities. A numerical procedure for computing the accessible fraction of the circle of intersection between two surface spheres is implemented in SASAD. The analytical method for surface area calculation proposed by Richmond (1984) also provides analytical gradients, and revised versions of this approach were implemented in molecular dynamics programs by Wesson and Eisenberg (1992) and Fraczkiewicz and Braun (1998). All existing methods for computing the derivatives are extensions of strategies used for computing the surface area, and therefore suffer the same stability problems. The Alpha Shapes software proposes a robust solution to the latter problem, by implementing arbitrary precision arithmetic to avoid numerical problems and systematically resolving all singularities without explicitly perturbing the positions of the sphere centers. The latter method is referred to as Simulation of Simplicity, as described by Edelsbrunner and Mücke (1990). In this paper we describe an extension of the Alpha Shapes method that includes the efficient, robust, exact, and analytical computation of the derivatives of surface area terms. There is an inherent difficulty in using a potential based on surface area for energy minimization or molecular dynamics. Although the accessible surface area is continuous in the position of the atoms, its derivatives are not. Wawak et al. (1994) have published a list of situations in which discontinuity in the derivative is observed. We re-examine this issue within the framework of the Alpha Shape method and relate discontinuities with combinatorial changes in the subcomplex of the Delaunay triangulation that is dual to the space-filling diagram.

Outline. Section 2 explains our approach to computing derivatives and states the results. Section 3 proves the formulas for the unweighted and the weighted area derivatives. Sections 4 and 5 discuss the continuity of the derivatives and the implementation of the algorithm. Section 6 concludes this paper.

\section{Approach and Results}

In this section we explain how we approach the problem of computing the derivatives of the surface area of a three-dimensional space-filling diagram.

Derivatives. We need some notation from vector calculus to talk about derivatives. We refer to Spivak (1965) for an introduction to that topic. For a differentiable map $f: \mathbb{R}^{m} \rightarrow \mathbb{R}$, the derivative at a point $\mathbf{z} \in \mathbb{R}^{m}$ is a linear map $\mathrm{D} f_{\mathbf{z}}: \mathbb{R}^{m} \rightarrow \mathbb{R}$. The 
geometric interpretation is as follows. The graph of $\mathrm{D} f_{\mathbf{z}}$ is the tangent space of $f$ at $\mathbf{z}$, which is a hyperplane passing through the origin in $\mathbb{R}^{m+1}$. The translation that moves the origin to the point $(\mathbf{z}, f(\mathbf{z}))$ on the graph of $f$ moves the tangent space to the supporting hyperplane at that point. Being linear, $\mathrm{D} f_{\mathbf{z}}$ can be written as the scalar product of the variable vector $\mathbf{t} \in \mathbb{R}^{m}$ with a fixed vector $\mathbf{u} \in \mathbb{R}^{m}$ known as the gradient of $f$ at $\mathbf{z}$ : $\mathrm{D} f_{\mathbf{z}}(\mathbf{t})=\langle\mathbf{u}, \mathbf{t}\rangle$. The derivative $\mathrm{D} f$ maps each $\mathbf{z} \in \mathbb{R}^{m}$ to $\mathrm{D} f_{\mathbf{z}}$ or, equivalently, to its gradient $\mathbf{u}$.

In this paper we call points in $\mathbb{R}^{m}$ states and use them to represent sets of balls in $\mathbb{R}^{3}$. For $m=3 n$, the state $\mathbf{z}$ represents the set of balls $B_{i}=\left(z_{i}, \varrho_{i}\right)$, for $0 \leq i \leq n-1$, where $\left[\mathbf{z}_{3 i+1}, \mathbf{z}_{3 i+2}, \mathbf{z}_{3 i+3}\right]^{T}=z_{i}$ is the center of $B_{i}$. The radius $\varrho_{i}$ is assumed to be fixed and is not encoded in the state. The weighted and unweighted areas of the union of the $B_{i}$ are maps $E, A: \mathbb{R}^{3 n} \rightarrow \mathbb{R}$. Their derivatives at a state $\mathbf{z} \in \mathbb{R}^{3 n}$ are linear maps $\mathrm{D} E_{\mathbf{z}}, \mathrm{D} A_{\mathbf{z}}: \mathbb{R}^{3 n} \rightarrow \mathbb{R}$, and the goal of this paper is to give a complete description of these derivatives.

Power Diagram. Let $S_{i}$ be the sphere bounding $B_{i}$. A useful concept is the power distance of a point $x$ from $S_{i}$, which is defined as $\pi_{i}(x)=\left\|x-z_{i}\right\|^{2}-\varrho_{i}^{2}$. Note that $S_{i}$ consists of all points $x$ with zero and $B_{i}$ of all points with non-positive power distance. The set of points with equal power distance from two spheres is their radical plane. It contains the circle common to both spheres, if it exists. The radical plane bounds the half-space of points whose power distance from one sphere is less than or equal to that from the other. Consider the collection of spheres $S_{i}$, for $0 \leq i \leq n-1$. The power cell of $S_{i}$ is the set of points $x$ for which $S_{i}$ minimizes the power distance,

$$
P_{i}=\left\{x \in \mathbb{R}^{3} \mid \pi_{i}(x) \leq \pi_{j}(x), \forall j\right\} .
$$

Each power cell is a convex polyhedron with at most $n-1$ polygonal faces. The polyhedra cover the entire $\mathbb{R}^{3}$ and have pairwise disjoint interiors. The power diagram consists of the power cells together with their polygonal faces, edges, and vertices. Let $F=\bigcup_{i} B_{i}$ be the space-filling diagram. If we restrict the power diagram to within $F$, we get a decomposition of $F$ into the convex cells $P_{i} \cap F=P_{i} \cap B_{i}$. Figure 1 illustrates this decomposition for the two-dimensional case.

Dual Complex. The (weighted) Delaunay triangulation is the dual of the power diagram. It is obtained by taking the sphere centers as vertices and drawing an edge between

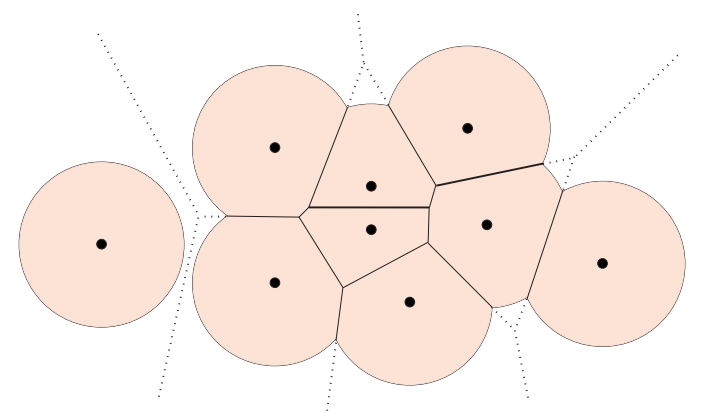

Fig. 1. The edges of the power diagram are solid inside and dotted outside the union of disks. 


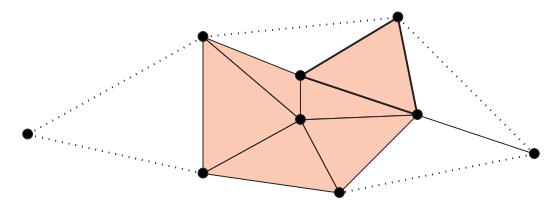

Fig. 2. The Delaunay triangulation and dual complex of the disks in Fig. 1. The dotted edges and unshaded triangles belong to the Delaunay triangulation, and the remaining simplices belong to both.

any two vertices whose corresponding power cells share a common polygonal face. We also draw a triangle for every three power cells that share an edge and a tetrahedron for every four power cells that share a vertex. It is convenient to assume or simulate general position, so that no other types of common intersections among power cells occur. In this case, all dual elements are simplices and the Delaunay triangulation is a simplicial complex. We write $\tau \leq v$ if the simplex $\tau$ is a face of the simplex $v$.

The dual complex $K$ is the dual of the decomposition of $F$. Its vertices are again the sphere centers, but it contains only those simplices that correspond to collections of clipped cells with non-empty common intersection. Since $P_{i} \cap B_{i} \subseteq P_{i}$, for every $i$, the dual complex is a subcomplex of the Delaunay triangulation. Figure 2 illustrates the definition by showing the Delaunay triangulation and the dual complex of the nine disks in Fig. 1.

We formalize the notion of neighborhood within $K$ by defining the star of a simplex $\tau \in K$ as the set of simplices that contain $\tau$. The link of $\tau$ is the collection of faces of simplices in the star that are disjoint from $\tau$. More formally,

$$
\begin{aligned}
\text { St } \tau & =\{v \in K \mid \tau \leq v\}, \\
\operatorname{Lk} \tau & =\{\omega \in K \mid \omega \leq v \in \operatorname{St} \tau \text { and } \omega \cap \tau=\emptyset\} .
\end{aligned}
$$

For example, the star of an interior edge contains the edge together with a ring of triangles and tetrahedra around the edge. The link is a cycle that consists of a vertex for each triangle and an edge for each tetrahedron in the star.

Measuring. We use fractions to express the size of geometric entities in the decomposition of the space-filling diagram. For example, $\sigma_{i}=\operatorname{area}\left(P_{i} \cap \operatorname{bd} F\right) / \operatorname{area}\left(S_{i}\right)$ is the fraction of the $i$ th sphere on the boundary of the space-filling diagram. The area of $F=\bigcup_{i} B_{i}$ is therefore $A=4 \pi \sum \varrho_{i}^{2} \sigma_{i}$. Given real weights $\alpha_{i}$, the weighted area is $E=4 \pi \sum \alpha_{i} \varrho_{i}^{2} \sigma_{i}$. If the $\alpha_{i}$ are the atomic solvation parameters, $E$ is also known as the solvation energy. The formulas for the derivatives require fractions of circles and line segments. Two spheres intersect in a possibly empty circle $S_{i j}=S_{i} \cap S_{j}$. The fraction that belongs to the boundary of the space-filling diagram is

$$
\sigma_{i j}=\frac{\text { length }\left(P_{i} \cap P_{j} \cap \text { bd } F\right)}{\text { length }\left(S_{i j}\right)} .
$$

Three spheres $S_{i}, S_{j}$, and $S_{k}$ intersect in a pair of points, and these points span a line segment $B_{i j k}$, if they exist. The fraction that belongs to the corresponding edge of the power diagram is

$$
\beta_{i j k}=\frac{\text { length }\left(P_{i} \cap P_{j} \cap P_{k} \cap F\right)}{\text { length }\left(B_{i j k}\right)} .
$$


Given the dual complex $K$, it is fairly straightforward to compute the $\sigma_{i}, \sigma_{i j}$, and $\beta_{i j k}$. We explain this for the fractions needed to express the derivatives. Let $S_{i j}^{k}$ be the portion of the circle $S_{i j}$ on $S_{k}$ 's side of the bisectors, $S_{i j}^{k}=\left\{x \in \mathbb{R}^{3} \mid \pi_{k}(x) \leq \pi_{j}(x)=\pi_{i}(x)=0\right\}$. The general results in Edelsbrunner (1995) imply the following inclusion-exclusion formula for this fraction:

$$
\sigma_{i j}=1-\frac{\sum_{k} \text { length }\left(S_{i j}^{k}\right)-\sum_{k, \ell} \text { length }\left(S_{i j}^{k} \cap S_{i j}^{\ell}\right)}{2 \pi \varrho_{i j}},
$$

where $\varrho_{i j}$ is the radius of $S_{i j}$. The first sum ranges over all vertices $z_{k}$ and the second over all edges $z_{k} z_{\ell}$ in the link of $z_{i} z_{j}$ in $K$. We note that $\sigma_{i j} \neq 0$ iff $z_{i} z_{j}$ is a boundary edge of the dual complex. Let $B_{i j k}^{\ell}$ be the portion of $B_{i j k}$ on $S_{\ell}$ 's side of the bisectors. Then

$$
\beta_{i j k}=1-\frac{\sum_{\ell} \text { length }\left(B_{i j k}^{\ell}\right)}{2 \varrho_{i j k}}
$$

where $2 \varrho_{i j k}=$ length $\left(B_{i j k}\right)$. Here the sum ranges over all vertices in the link of the triangle $z_{i} z_{j} z_{k}$ in $K$. There are at most two such vertices, each clipping one side of the line segment to get the corresponding edge in the power diagram.

Results. The first result of this paper is a complete description of the area derivative. Let $\zeta_{i j}=\left\|z_{i}-z_{j}\right\|$ be the distance between two centers. We write $u_{i j}=\left(z_{i}-z_{j}\right) / \zeta_{i j}$ for the unit vector in the direction of the connecting line. For each $k \neq i, j$, let $v_{i j k}=$ $u_{i k}-\left\langle u_{i k}, u_{i j}\right\rangle \cdot u_{i j}$ be the component of $u_{i k}$ normal to $u_{i j}$, and let $u_{i j k}=v_{i j k} /\left\|v_{i j k}\right\|$ be the unit vector in that normal direction.

Area Derivative Theorem. The derivative of the area of the space-filling diagram with state $\mathbf{z} \in \mathbb{R}^{3 n}$ is $\mathrm{D} A_{\mathbf{z}}(\mathbf{t})=\langle\mathbf{a}, \mathbf{t}\rangle$, where

$$
\begin{aligned}
{\left[\begin{array}{l}
\mathbf{a}_{3 i+1} \\
\mathbf{a}_{3 i+2} \\
\mathbf{a}_{3 i+3}
\end{array}\right] } & =\sum_{j}\left(\sigma_{i j} \cdot a_{i j}+\sum_{k} \beta_{i j k} \cdot a_{i j k}\right), \\
a_{i j} & =\pi\left(\varrho_{i}+\varrho_{j}\right)\left[1-\frac{\left(\varrho_{i}-\varrho_{j}\right)^{2}}{\zeta_{i j}^{2}}\right] \cdot u_{i j}, \\
a_{i j k} & =2 \varrho_{i j k} \frac{\varrho_{i}-\varrho_{j}}{\zeta_{i j}} \cdot u_{i j k},
\end{aligned}
$$

for $0 \leq i<n$. The summation is over all boundary edges $z_{i} z_{j}$ and their triangles $z_{i} z_{j} z_{k}$ in $K$.

The second result is a complete description of the weighted area derivative. Let $\alpha_{i} \in \mathbb{R}$ be the weight of the $i$ th sphere. 
Weighted Area Derivative Theorem. The derivative of the weighted area of the space-filling diagram with state $\mathbf{z} \in \mathbb{R}^{3 n}$ is $\mathrm{D} E_{\mathbf{z}}(\mathbf{t})=\langle\mathbf{e}, \mathbf{t}\rangle$, where

$$
\begin{aligned}
{\left[\begin{array}{l}
\mathbf{e}_{3 i+1} \\
\mathbf{e}_{3 i+2} \\
\mathbf{e}_{3 i+3}
\end{array}\right] } & =\sum_{j}\left(\sigma_{i j} \cdot e_{i j}+\sum_{k} \beta_{i j k} \cdot e_{i j k}\right), \\
e_{i j} & =\pi\left[\left(\alpha_{i} \varrho_{i}+\alpha_{j} \varrho_{j}\right)-\left(\alpha_{i} \varrho_{i}-\alpha_{j} \varrho_{j}\right) \frac{\varrho_{i}^{2}-\varrho_{j}^{2}}{\zeta_{i j}^{2}}\right] \cdot u_{i j}, \\
e_{i j k} & =2 \varrho_{i j k} \frac{\alpha_{i} \varrho_{i}-\alpha_{j} \varrho_{j}}{\zeta_{i j}} \cdot u_{i j k},
\end{aligned}
$$

for $0 \leq i<n$. The summation is over all boundary edges $z_{i} z_{j}$ and their triangles $z_{i} z_{j} z_{k}$ in $K$.

We note that $\mathrm{D} A$ and $\mathrm{D} E$ are not everywhere continuous. Specifically, they may be discontinuous when two spheres touch, three spheres intersect in a common circle, or four spheres intersect in the same two points.

\section{Derivation}

This section derives the formulas claimed in the two theorems. They are the sums of contributions from locally direction preserving and distance preserving components of the motion. A direction is determined by two spheres and preserved if they move on the straight line that passes through their centers. A distance preserving motion is a rotation of one sphere about another.

Direction Preserving Motion. To study the derivative for a direction preserving motion, we define $F=B_{i} \cup B_{j}$ and assume the two bounding spheres, $S_{i}$ and $S_{j}$, have a nonempty intersection. Let $\zeta_{i}$ and $\zeta_{j}$ be the signed distances of $z_{i}$ and $z_{j}$ from the radical plane, as illustrated in Fig. 3. The sum of the two distances is $\zeta_{i j}=\zeta_{i}+\zeta_{j}=\left\|z_{i}-z_{j}\right\|$. We have $\varrho_{i}^{2}-\varrho_{j}^{2}=\zeta_{i}^{2}-\zeta_{j}^{2}=\zeta_{i j}\left(\zeta_{i}-\zeta_{j}\right)$ and therefore

$$
\zeta_{i}=\frac{1}{2}\left(\zeta_{i j}+\frac{\varrho_{i}^{2}-\varrho_{j}^{2}}{\zeta_{i j}}\right),
$$

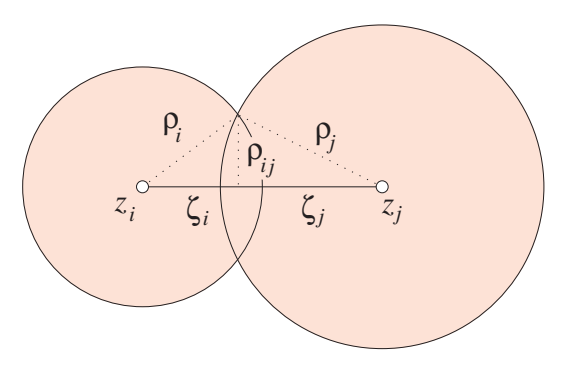

Fig. 3. The two spheres intersect in a circle with radius $\varrho_{i j}$. 


$$
\zeta_{j}=\frac{1}{2}\left(\zeta_{i j}+\frac{\varrho_{j}^{2}-\varrho_{i}^{2}}{\zeta_{i j}}\right) .
$$

Using Archimedes' area formula for spheres, we get

$$
\begin{aligned}
A & =4 \pi \varrho_{i}^{2} \frac{\varrho_{i}+\zeta_{i}}{2 \varrho_{i}}+4 \pi \varrho_{j}^{2} \frac{\varrho_{j}+\zeta_{j}}{2 \varrho_{j}} \\
& =2 \pi\left(\varrho_{i}^{2}+\varrho_{j}^{2}\right)+\pi\left(\varrho_{i}+\varrho_{j}\right)\left[\zeta_{i j}+\frac{\left(\varrho_{i}-\varrho_{j}\right)^{2}}{\zeta_{i j}}\right],
\end{aligned}
$$

for the area of the boundary of $F$, where the second line is derived from the first by a few simple algebraic manipulations using (1) and (2). The equation for $A$ is valid as long as the two spheres have a non-empty intersection, or equivalently as long as $\left|\varrho_{i}-\varrho_{j}\right| \leq \zeta_{i j} \leq \varrho_{i}+\varrho_{j}$. The derivative with respect to the distance between the two centers is therefore

$$
\frac{\mathrm{d} A}{\mathrm{~d} \zeta_{i j}}=\pi\left(\varrho_{i}+\varrho_{j}\right)\left[1-\frac{\left(\varrho_{i}-\varrho_{j}\right)^{2}}{\zeta_{i j}^{2}}\right] .
$$

Figure 4 sketches the function within the interval of permitted distances.

Distance Preserving Motion. To study the area derivative for a distance preserving motion, we define $F=B_{i} \cup B_{j} \cup B_{k}$ and assume the three bounding spheres intersect in two points, $\{p, q\}=S_{i} \cap S_{j} \cap S_{k}$. Recall that $B_{i j k}$ is the line segment with endpoints $p$ and $q$. In the assumed case, the two caps $C_{i j}=B_{i} \cap S_{j}$ and $C_{k j}=B_{k} \cap S_{j}$ have a non-empty common intersection, and their bounding circles intersect in the same two points, $\{p, q\}=S_{i j} \cap S_{k j}$, as shown in Fig. 5. The spherical distance between the centers $y_{i}$ and $y_{k}$ of the two caps is $\eta_{j}=\varrho_{j} \cdot \angle y_{i} z_{j} y_{k}$. Consider the area of the union, $A_{j}=\operatorname{area}\left(C_{i j} \cup C_{k j}\right)$. We use geometric reasoning to compute the derivative with respect to the spherical distance. To the first order, the area gained by moving the two caps apart, while keeping their centers on the same great-circle, is equal to the area of a spherical rectangle. Its height is the spherical distance between $p$ and $q$ and its width is $\mathrm{d} \eta_{j}$. Using Archimedes' formula, the area of the slice of $S_{j}$ between the two planes

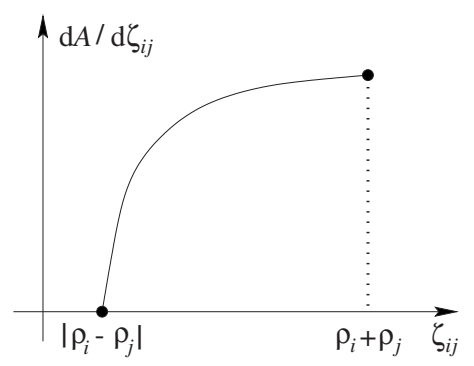

(a)

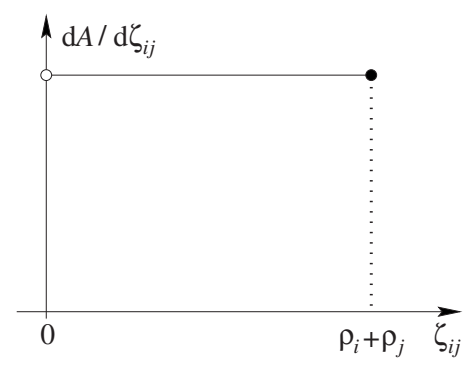

(b)

Fig. 4. (a) The graph for $\varrho_{i} \neq \varrho_{j}$, in which case the area derivative is positive at $\zeta_{i j}=\varrho_{i}+\varrho_{j}$ and zero at $\zeta_{i j}=\left|\varrho_{i}-\varrho_{j}\right|$. (b) The graph for $\varrho_{i}=\varrho_{j}$, in which case the area derivative is a positive constant over the entire interval, except for $\zeta_{i j}=0$, where it is undefined. 


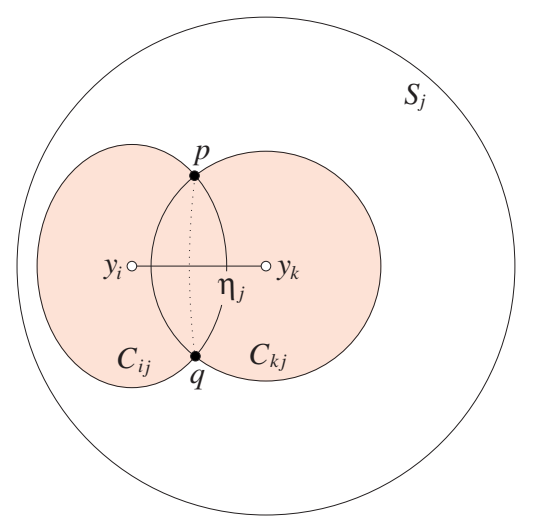

Fig. 5. Two spherical caps whose bounding circles intersect in two points.

passing through $p$ and $q$ normal to $p q$ is the area of $S_{j}$ times the Euclidean distance between $p$ and $q$ divided by the diameter, which is $4 \pi \varrho_{j}^{2}\|p-q\| / 2 \varrho_{j}=4 \pi \varrho_{j} \varrho_{i j k}$. The area of the rectangle is the area of the slice times $\mathrm{d} \eta_{j}$ divided by $2 \pi \varrho_{j}$. The derivative of the area covered by the two caps is therefore

$$
\frac{\mathrm{d} A_{j}}{\mathrm{~d} \eta_{j}}=2 \varrho_{i j k} .
$$

Figure 6 sketches the function within the interval of permitted spherical distances.

Assembling the Relations. Let $\mathbf{t}$ be the velocity vector of the motion at state $\mathbf{z}$. Then $t_{i}=\left[\mathbf{t}_{3 i+1}, \mathbf{t}_{3 i+2}, \mathbf{t}_{3 i+3}\right]^{T}$ is the velocity vector of $z_{i}$. Because the derivative is linear, we can decompose the motion into components and add the contributions. For every ordered pair $i, j$, we consider the direction preserving component $\left\langle u_{i j}, t_{i}\right\rangle \cdot u_{i j}$, and for every ordered triplet $i, j, k$, we consider the distance preserving component $\left\langle u_{i j k}, t_{i}\right\rangle \cdot u_{i j k}$ of $t_{i}$.

The contribution of the ordered pair $i, j$ is the fraction of the circle $S_{i j}$ that belongs to the boundary of $F=\bigcup_{i} B_{i}$ times what is given in (3). This is $\sigma_{i j}\left\langle a_{i j}, t_{i}\right\rangle$, where $a_{i j}$ is as

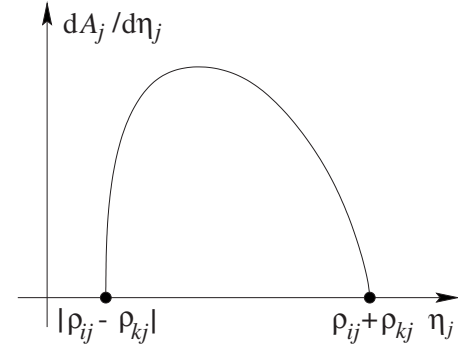

(a)

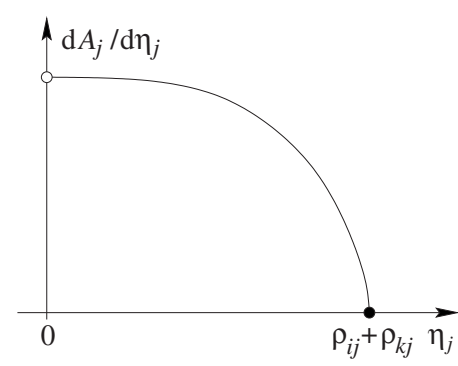

(b)

Fig. 6. (a) The graph for $\varrho_{i j} \neq \varrho_{k j}$, in which case the area derivative at both extremes is zero with infinite slope. (b) The graph for $\varrho_{i j}=\varrho_{k j}$, in which case the derivative approaches twice the common radius as the distance between the two centers goes to zero. 
given in the Area Derivative Theorem. One part of the contribution of the ordered triplet $i, j, k$ is the fraction of the line segment $B_{i j k}$, that belongs to the corresponding edge of the power diagram, times the negative of what is given in (4). We take the negative because the two measured caps subtract from the area of $F$. The other part of the contribution of $i, j, k$ is the same fraction of $B_{i j k}$ times what is given in (4) after switching $i$ and $j$ (which does not change the right side of the equation). This part accounts for the two caps $C_{j i}$ and $C_{k i}$ on $S_{i}$ and is taken positive because the motion of $S_{i}$ along $t_{i}$ with fixed $S_{j}$ is relatively the same as the motion of $S_{j}$ along $-t_{i}$ with fixed $S_{i}$. The velocity vector of the center of the cap $C_{i j}$ is $\varrho_{j} / \zeta_{i j}$ times the component of $t_{i}$ along $u_{i j k}$, and that of the center of the cap $C_{j i}$ in the relative motion is $-\varrho_{i} / \zeta_{i j}$ times the same vector. The contribution of $i, j, k$ to the area derivative is therefore $\beta_{i j k}\left\langle a_{i j k}, t_{i}\right\rangle$, where $a_{i j k}$ is as given in the Area Derivative Theorem. Adding the terms for all ordered pairs and triplets gives

$$
\mathrm{D} A_{\mathbf{z}}(\mathbf{t})=\sum_{i=0}^{n-1} \sum_{j \neq i}\left(\sigma_{i j}\left\langle a_{i j}, t_{i}\right\rangle+\sum_{k \neq i, j} \beta_{i j k}\left\langle a_{i j k}, t_{i}\right\rangle\right)
$$

for the area derivative. We can write this more succinctly as $\mathrm{D} A_{\mathbf{z}}(\mathbf{t})=\langle\mathbf{a}, \mathbf{t}\rangle$, where $\mathbf{a}$ is as defined in the Area Derivative Theorem. We have $\sigma_{i j}=0$ unless $z_{i} z_{j}$ is a boundary edge and $\beta_{i j k}=0$ unless $z_{i} z_{j} z_{k}$ is a triangle of $K$. Furthermore, the sum over indices $k$ vanishes unless $z_{i} z_{j}$ is a boundary edge. This implies that the summation can be limited to all boundary edges $z_{i} z_{j}$ and their triangles $z_{i} z_{j} z_{k}$ in $K$, as stated in the Area Derivative Theorem.

The Weighted Case. Let $\alpha_{i}$ be a real number for $0 \leq i<n$, and define the weighted area of the space-filling diagram $F$ equal to

$$
E=4 \pi \sum_{i=0}^{n-1} \alpha_{i} \varrho_{i}^{2} \sigma_{i}
$$

where $\sigma_{i}$ is the fraction of the $i$ th sphere that belongs to the boundary of $F$. Similar to the unweighted case, we distinguish between direction and distance preserving components of the motion. We get the derivatives by straightforward modifications of the unweighted formulas. For the direction preserving motion, we consider again the union of two balls whose bounding spheres intersect in a common circle, $F=B_{i} \cup B_{j}$. Using Archimedes' area formula for spheres, we get

$$
\begin{aligned}
E & =4 \pi \alpha_{i} \varrho_{i}^{2} \frac{\varrho_{i}+\zeta_{i}}{2 \varrho_{i}}+4 \pi \alpha_{j} \varrho_{j}^{2} \frac{\varrho_{j}+\zeta_{j}}{2 \varrho_{j}} \\
& =2 \pi\left(\alpha_{i} \varrho_{i}^{2}+\alpha_{j} \varrho_{j}^{2}\right)+\pi\left(\alpha_{i} \varrho_{i}+\alpha_{j} \varrho_{j}\right) \zeta_{i j}+\pi\left(\alpha_{i} \varrho_{i}-\alpha_{j} \varrho_{j}\right) \frac{\varrho_{i}^{2}-\varrho_{j}^{2}}{\zeta_{i j}} .
\end{aligned}
$$

The derivative with respect to the distance between the centers is

$$
\frac{\mathrm{d} E}{\mathrm{~d} \zeta_{i j}}=\pi\left(\alpha_{i} \varrho_{i}+\alpha_{j} \varrho_{j}\right)-\pi\left(\alpha_{i} \varrho_{i}-\alpha_{j} \varrho_{j}\right) \frac{\varrho_{i}^{2}-\varrho_{j}^{2}}{\zeta_{i j}^{2}},
$$


which for $\alpha_{i}=\alpha_{j}=1$ agrees with (3). For a distance preserving motion, we generalize (4) to

$$
\frac{\mathrm{d} E_{j}}{\mathrm{~d} \eta_{j}}=2 \alpha_{j} \varrho_{i j k} .
$$

Assembling these relations as in the unweighted case, we get

$$
\mathrm{D} E_{\mathbf{z}}(\mathbf{t})=\sum_{i=0}^{n-1} \sum_{j \neq i}\left(\sigma_{i j}\left\langle e_{i j}, t_{i}\right\rangle+\sum_{k \neq i, j} \beta_{i j k}\left\langle e_{i j k}, t_{i}\right\rangle\right)
$$

for the weighted area derivative. We can write this more succinctly as $\mathrm{D} E_{\mathbf{z}}(\mathbf{t})=\langle\mathbf{e}, \mathbf{t}\rangle$, where $e_{i j}, e_{i j k}$, and $\mathbf{e}$ is as defined in the Weighted Area Derivative Theorem. Similar to the unweighted case, the summation can be limited to all boundary edges $z_{i} z_{j}$ and their triangles $z_{i} z_{j} z_{k}$ in $K$.

\section{Discontinuity}

In this section we enumerate the cases in which the area derivative can be discontinuous and relate them to singularities in the definition of the dual complex. Since the weighted area contains the unweighted area as a special case, we may restrict the discussion to the former.

Combination of Maps. Recall that the derivative of the weighted area function is the map $\mathrm{D} E: \mathbb{R}^{3 n} \rightarrow \mathbb{R}^{3 n}$ defined by $\mathrm{D} E(\mathbf{z})=\mathbf{e}$. It has discontinuities along a measure zero subset of $\mathbb{R}^{3 n}$, where $E$ is not differentiable. This subset has been studied before in the context of molecular dynamics by Wawak et al. (1994) and can be understood by examining the formula in the Weighted Area Derivative Theorem. That formula is composed of the fraction maps $\sigma_{i j}$ and $\beta_{i j k}$, the coefficient maps $\left\|e_{i j}\right\|$ and $\left\|e_{i j k}\right\|$, and the direction maps $u_{i j}$ and $u_{i j k}$. All six are almost everywhere but not everywhere continuous. We have a discontinuity of $\mathrm{D} E$ only if at least one of the maps is discontinuous and the multiplying other maps are non-zero. Note, however, that having one discontinuous map with a non-zero multiplying factor does not necessarily imply a discontinuity since there may be relations that cause non-trivial cancellations.

We will see shortly that every state $\mathbf{z}$ at which $\mathrm{D} E$ is discontinuous also has the property that every open neighborhood contains states with combinatorially different dual complexes. In other words, when $\mathbf{z}$ passes through a discontinuity of $\mathrm{D} E$ then the dual complex passes a moment at which it may undergo a combinatorial change. All these changes happen at the boundary of the dual complex, but not every change on the boundary corresponds to a discontinuity.

Pair Maps. We first consider the maps that depend on two spheres, $\sigma_{i j}$, $\left\|e_{i j}\right\|$, and $u_{i j}$. The three cases in which $\sigma_{i j}$ may be discontinuous are illustrated in Fig. 7. Case (b) is further split into Case (b.1), when $\varrho_{i} \neq \varrho_{j}$, and Case (b.2), when $\varrho_{i}=\varrho_{j}$.

Case (a). Spheres $S_{i}$ and $S_{j}$ touch in a point $x$ and lie on opposite sides of the common tangent plane. The states that satisfy this description form a $(3 n-1)$-dimensional cylinder 


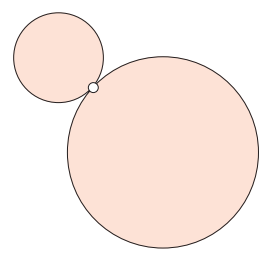

(a)

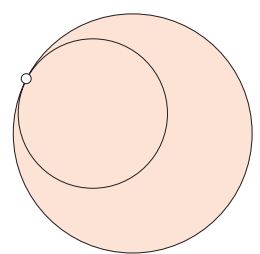

(b)

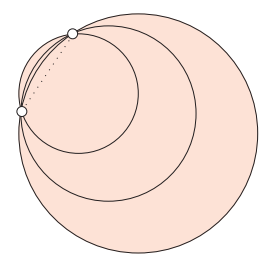

(c)

Fig. 7. (a) Two non-nested touching spheres. (b) Two nested touching spheres. (c) Three spheres meeting in a common circle.

in $\mathbb{R}^{3 n}$. If $x$ lies outside all other spheres, then $\sigma_{i j}$ jumps from 0 to 1 or from 1 to 0 . The maps $\left\|e_{i j}\right\|$ and $u_{i j}$ may both be non-zero, which implies that $\mathrm{D} E$ can be discontinuous.

Case (b.1). Spheres $S_{i}$ and $S_{j}$ touch in a point $x$, they lie on the same side of the common tangent plane, and $\varrho_{i} \neq \varrho_{j}$. As in Case (a), the states that satisfy this condition form a $(3 n-1)$-dimensional cylinder in $\mathbb{R}^{3 n}$, and if $x$ lies outside all other balls, then $\sigma_{i j}$ jumps from 0 to 1 or from 1 to 0 . In the unweighted case we have $\left\|a_{i j}\right\|=0$, as shown in Fig. 4(a), and therefore no discontinuity of the derivative. In the weighted case we may have $\left\|e_{i j}\right\| \neq 0$, and since $u_{i j}$ is non-zero by construction, $\mathrm{D} E$ can be discontinuous.

Case (b.2): $S_{i}=S_{j}$. The states that satisfy this description form a (3n-3)-dimensional linear subspace of $\mathbb{R}^{3 n}$. Using limit considerations, we get a possibly non-zero continuous extension for the pairwise coefficient map, namely $\left\|e_{i j}\right\|=\pi\left(\alpha_{i} \varrho_{i}+\alpha_{j} \varrho_{j}\right)$. If a nonzero fraction of the common sphere surface lies on the boundary of $F$, then we also get non-zero limits for $\sigma_{i j}$. Since there is no continuous extension of the direction map, DE can be discontinuous.

Case (c). Spheres $S_{i}, S_{j}$, and $S_{k}$ intersect in a common circle. The states that satisfy this description form a $(3 n-3)$-dimensional subset of $\mathbb{R}^{3 n}$. If a positive fraction of the common circle belongs to the boundary of $F$, we get positive limits for $\sigma_{i j}, \sigma_{i k}$, and $\sigma_{j k}$, which are different for different approaching directions. The coefficient maps and direction maps can all be non-zero, which implies that $\mathrm{D} E$ can be discontinuous.

The state can pass through a singularity in various ways. The generic way of passing through Cases (a) and (b.1) corresponds to adding an edge to the dual complex or removing it from the same. The generic way of passing through Case (b.2) corresponds to contracting an edge followed by un-contracting the same. There is more than one way to generically pass through Case (c), and in each way we witness the removal of a degree- 2 vertex and the replacement of its two edges by a new edge connecting their two other endpoints, or the inverse of that operation.

Triple Maps. The maps $\beta_{i j k},\left\|e_{i j k}\right\|$, and $u_{i j k}$ have discontinuities where the circles bounding caps on $S_{j}$ form configurations as in Fig. 7.

Case (d). The spheres $S_{i}, S_{j}$, and $S_{k}$ intersect in a common point, as in Fig. 7(a),(b). In this case we have $\varrho_{i j k}=0$. The discontinuity of $\beta_{i j k}$ is thus wiped out by the vanishing of $\left\|e_{i j k}\right\|$, which implies that $\mathrm{D} E$ is continuous. 
Case (e). The spheres $S_{i}, S_{j}, S_{k}$, and $S_{\ell}$ intersect in a common point pair, as in Fig. 7(c). The states that satisfy this description form a $(3 n-2)$-dimensional cylinder in $\mathbb{R}^{3 n}$. We can arrange the other spheres so we get positive limits for $\beta_{i j k}, \beta_{i j \ell}, \beta_{i k \ell}$, and $\beta_{j k \ell}$ that are different for different approaching directions. We can choose the centers and radii such that the coefficient maps and direction maps are all non-zero. It thus seems possible that $\mathrm{D} E$ is discontinuous.

The generic way of passing through Case (d) corresponds to adding a triangle to the dual complex or removing it from the same. This triangle may fill or open a tunnel, or it may be added or removed together with one of its edges. Similarly, the generic way of passing through Case (e) corresponds to adding a tetrahedron to the dual complex or removing it from the same. The tetrahedron may fill or open a void, or it may be added or removed together with one of its triangles.

If no Cases (a)-(e) occur in the collection of spheres defining the space-filling diagram, then we have continuous fraction maps, continuous coefficient maps, continuous direction maps, and therefore a state where the weighted area derivative is continuous.

\section{Implementation}

We implemented the Weighted Area Derivative Theorem by re-implementing the Alpha Shapes software to compute the dual complex of the space-filling diagram. As described below, we have validated the resulting software as correct and sufficiently fast for applications in molecular dynamics.

Molecular Simulation Application. Implicit solvent models usually compute the interaction of water with non-polar atoms of a molecule as a weighted sum of the accessible surface area of each atom. The weights are the atomic solvation parameters introduced by Eisenberg and McLachlan (1986). These terms, and their derivatives with respect to the position of the atoms, can be computed using the Alpha Shape software and the Weighted Area Derivative Theorem with the coefficient $\alpha_{i}$ set to the values of the atomic solvation parameters. Applications of this method to molecular simulations such as molecular energy minimization or molecular dynamics require that special care be taken to minimize the computational cost. One step of a molecular dynamics simulation in vacuo of a 90-residue protein requires approximately 40 milliseconds (ms) on a 1000 MHz Pentium III computer; the same simulation performed with explicit water requires $240 \mathrm{~ms}$. An implicit solvent simulation should therefore position itself between these two figures. We have written a new version of the Alpha Shape software, ALPHAMOL, specific to molecular simulation applications that includes implementations of the area and weighted area derivative formulas. Computation of the surface area and its derivatives of a 90-residue protein using ALPHAMOL require $60 \mathrm{~ms}$ on a $1000 \mathrm{MHz}$ Pentium III computer, which is well within the specifications required for molecular dynamics. The $60 \mathrm{~ms}$ roughly break down to $20 \mathrm{~ms}$ for computing the Delaunay triangulation, 20 $\mathrm{ms}$ for generating the alpha complex, $10 \mathrm{~ms}$ for computing the weighted surface area, and $10 \mathrm{~ms}$ to compute the weighted surface area derivatives.

The surface area is involved in the non-polar part, $W_{\text {np }}$, of the effective solvation potential $W$. The contribution of solvent to the electrostatic of the protein, $W_{\text {elec }}$, is usually 
computed based on a generalized born model (Still et al., 1990). Fast implementation of the electrostatics under this model are currently four times slower than the corresponding in vacuo calculations (Im et al., 2003). One step of an implicit solvent molecular dynamics simulation of a 90-residue protein would therefore require approximately $220 \mathrm{~ms}$, which is similar to the time required for an explicit solvent simulation. Note that most of this computing time is now spent in evaluating the electrostatics contribution to the energy, where significant speed-up is expected in the near future (Im et al., 2003).

Validation. The area derivative formula was tested for 7PCY (99-residue protein, 871 atoms) and 1TIM (247-residue protein, 1870 atoms) against the numerically estimated first derivatives of surface area:

$$
\Delta \mathbf{A}_{3 i+j}=\frac{A\left(\mathbf{z}_{3 i+j}+\delta\right)-A\left(\mathbf{z}_{3 i+j}-\delta\right)}{2 \delta},
$$

where $\mathbf{z}_{3 i+j}$ is the $j$ th coordinate of the $i$ th atom center. A similar expression holds for the weighted area by replacing $A$ with $E$ in (7). The average relative differences between analytical and numerical derivatives were computed as a relative RMS error:

$$
\mu(A)=\frac{\sqrt{\sum_{i=0}^{n-1} \sum_{j=1}^{3}\left(\mathbf{a}_{3 i+j}-\Delta \mathbf{A}_{3 i+j}\right)^{2}}}{\sqrt{\sum_{i=0}^{n-1} \sum_{j=1}^{3}\left(\Delta \mathbf{A}_{3 i+j}\right)^{2}}},
$$

where $n$ is the number of atoms, as before. The average relative differences $\mu(E)$ for the weighted areas are computed in a similar way. For the weighted areas, the tests were performed using the atomic solvation parameters from Table 3 of Wesson and Eisenberg (1992). Using $\delta=0.0001$, we find $\mu(A)$ equal to $5.3 \cdot 10^{-8}$ and $5.4 \cdot 10^{-8}$ for 7PCY and $1 T I M$, respectively, and $\mu(E)$ equal to $5.6 \cdot 10^{-8}$ and $5.7 \cdot 10^{-8}$ for the same proteins. The good agreements between the analytical and numerical derivatives of the surface area and weighted surface areas show that our approach and implementation are correct.

Performance. We compared the performance of ALPHAMOL with that of one other analytical program for computing molecular surface area and its gradients, GETAREA by Fraczkiewicz and Braun (1998), and of one fast approximate program, PROSURF. The latter software is our own implementation of the fast numerical procedure proposed by LeGrand and Merz (1993). Accessible surface areas and their derivatives were calculated for a set of four proteins, 6PTI, 7PCY, 1TIM, and 1MCP. Atomic radii and solvation

Table 1. Comparison between AlphaMol, GetArea and PROSURF. The running time is given in seconds.

\begin{tabular}{lrrrr}
\hline & \multicolumn{4}{c}{ Protein } \\
\cline { 2 - 5 } & 6PTI & 7PCY & 1 1TIM & $1 \mathrm{MCP}$ \\
\hline Number of atoms & 444 & 871 & 1870 & 3401 \\
\hline ALPHAMOL & 0.04 & 0.06 & 0.24 & 0.47 \\
GETAREA & 0.05 & 0.11 & 0.26 & 0.48 \\
PROSURF & 0.03 & 0.06 & 0.17 & 0.32 \\
\hline
\end{tabular}


parameters were the same for every input protein. ALPHAMOL and GETAREA agree within numerical precision for both the surface area and derivatives, while the value of the surface area computed with PROSURF is found to be on average 3\% larger than the analytical values. Computing times on an Intel $1000 \mathrm{MHz}$ Pentium III computer for the three pieces of software are given in Table 5. ALPHAMOL is found to be slightly faster than GETAREA, and slightly slower than the numerical method. It should be mentioned however that the latter does not include the computation of the derivatives.

\section{Discussion}

The Alpha Shape software with the Weighted Area Derivative Theorem provides a fast, accurate, and robust method for computing the interaction of water with non-polar atoms of a molecule in an implicit solvent model. The corresponding software, ALPHAMOL, has similar CPU time requirements as other analytical or fast approximate programs. It surpasses numerical programs, however, in that it is accurate. To our knowledge, ALPHAMOL is also the only analytical program that explicitly deals with the problem of discontinuities of the weighted area derivatives. We have made preliminary steps towards inserting ALPHAMOL into the molecular dynamics program ENCAD by Levitt et al. (1995), but it is too early to say anything about the corresponding results.

When we evaluate the derivative formulas, we assume the availability of the dual complex of the space-filling diagram. At this time, we re-compute the entire Delaunay triangulation and obtain the dual complex by selecting its simplices from that triangulation. It would be worthwhile to develop a fast algorithm that maintains the dual complex during a continuous motion without repeatedly rebuilding the Delaunay triangulation.

The mathematical tools used in this paper to express the area derivative of a spacefilling diagram should prove useful to extend the results to the weighted volume derivatives and to the second derivatives of the weighted area and volume. It might also be interesting to compute derivatives for other molecular models. For example, the area derivative of the molecular skin surface defined by Edelsbrunner (1999) may turn out to be continuous and thus lead to more robust simulations than the partially discontinuous area derivative of the space-filling diagram.

\section{References}

Connolly, M. Analytical molecular surface calculation. J. Appl. Cryst. 16 (1983), 548-558.

Davis, M. E. The inducible multipole solvation model: a new model for solvation effects on solute electrostatics. J. Chem. Phys. 100 (1994), 5149-5159.

Edelsbrunner, H. The union of balls and its dual shape. Discrete Comput. Geom. 13 (1995), 415-440.

Edelsbrunner, H. Deformable smooth surface design. Discrete Comput. Geom. 21 (1999), 87-115.

Edelsbrunner, H., and Mücke, E. P. Simulation of simplicity: a technique to cope with degenerate cases in geometric algorithms. ACM Trans. Graphics 9 (1990), 66-104.

Eisenberg, D., and McLachlan, A. Solvation energy in protein folding and binding. Nature (London) 319 (1986), 199-203.

Eisenhaber, F., and Argos, P. Improved strategy in analytic surface calculation for molecular systems: handling of singularities and computational efficiency. J. Comput. Chem. 14 (1993), 1272-1280.

Fraczkiewicz, R., and Braun, W. Exact and efficient analytical calculation of the accessible surface area and their gradient for macromolecules. J. Comput. Chem. 19 (1998), 319-333. 
Fraternali, F., and Van Gunsteren, W. F. An efficient mean solvation force model for use in molecular dynamics simulations of proteins in aqueous solution. J. Mol. Biol. 256 (1996), 939-948.

Von Freyberg, B., Richmond, T. J., and Braun, W. Surface-area included in energy refinement of proteins: a comparative study on atomic solvation parameters. J. Mol. Biol. 233 (1993), 275-292.

Gibson, K., and Scheraga, H. Exact calculation of the volume and surface area of fused hard sphere molecules with unequal atomic radii. Molecular Phys. 62 (1987), 1247-1265.

Gogonea, V., and Osawa, E. An improved algorithm for the analytical computation of solvent-excluded volume. The treatment of singularities in solvent accessible surface area and volume functions. J. Comput. Chem. 7 (1995), 817-842.

Hasel, W., Hendrikson, T. F., and Still, W. C. A rapid approximation to the solvent accessible surface areas of atoms. Tetrahed. Comput. Method. 1 (1988), 103-116.

Im, W., Lee, M. S., and Brooks, C. L. Generalized born model with a simple smoothing function. J. Comput. Chem. 24 (2003), 1691-1702.

Kratky, K. W. The area of intersection of $n$ equal circular disks. J. Phys. A 11 (1978), 1017-1024.

Lee, B., and Richards, F. M. The interpretation of protein structure: estimation of static accessibility. J. Mol. Biol. 55 (1971), 379-400.

LeGrand, S. M., and Merz, K. M. Rapid approximation to molecular surface area via the use of Boolean logic and lookup tables. J. Comput. Chem. 14 (1993), 349-352.

Levitt, M., Hirshberg, M., Sharon, R., and Daggett, V. Potential-energy function and parameters for simulations of the molecular-dynamics of proteins and nucleic-acids in solution. Comput. Phys. Comm. 91 (1995), 215231 .

Liang, J., Edelsbrunner, H., Fu, P., Sudhakar, P. V., and Subramaniam, S. Analytical shape computation of macromolecules, I and II. Proteins: Struct. Funct. Genet. 33 (1998), 1-17 and 18-29.

Perrot, G., Cheng, B., Gibson, K. D., Vila, J., Palmer, K. A., Nayeem, A., Maigret, B., and Scheraga, H. A. MSEED: a program for the rapid analytical determination of accessible surface-areas and their derivatives. J. Comput. Chem. 13 (1992), 1-11.

Richmond, T. J. Solvent accessible surface area and excluded volume in proteins. J. Mol. Biol. 178 (1984), 63-89.

Schaefer, M., and Karplus, M. A comprehensive analytical treatment of continuum electrostatics. J. Phys. Chem. 100 (1996), 1578-1599.

Shrake, A., and Rupley, J. A. Environment and exposure to solvent of protein atoms in lyzozyme and insulin. J. Mol. Biol. 79 (1973), 351-371.

Spivak, M. Calculus on Manifolds. Addison-Wesley, Reading, Massachusetts, 1965.

Sridharan, S., Nicholls, A., and Sharp, K. A. A rapid method for calculating derivatives of solvent accessible surface areas of molecules. J. Comput. Chem. 16 (1994), 1038-1044.

Still, W. C., Tempczyk, A., Hawley, R. C., and Hendrickson, T. Semi-analytical treatment of solvation for molecular mechanics and dynamics. J. Amer. Chem. Soc. 112 (1990), 6127-6129.

Street, A. G., and Mayo, S. L. Pairwise calculation of protein solvent-accessible surface areas. Folding Design 3 (1998), 253-258.

Wawak, R. J., Gibson, K. D., and Scheraga, H. A. Gradient discontinuities in calculations involving molecular surface area. J. Math. Chem. 15 (1994), 207-232.

Wesson, L., and Eisenberg, D. Atomic solvation parameters applied to molecular dynamics of proteins in solution. Protein Sci. 1 (1992), 227-235.

Wodak, S. J., and Janin, J. Analytical approximation to the accessible surface area of proteins. Proc. Nat. Acad. Sci. U.S.A. 77 (1979), 1736-1740.

Received June 28, 2002. Online publication June 4, 2004. 